\title{
Études/Inuit/Studies
}

HOFFECKER, John F., 2005 A prehistory of the North. Human settlement of the higher latitude. Foreword by Brian Fagan, Rutgers University Press, New Brunswick, New Jersey, and London, 225 pages.

\section{Patrick Plumet}

Volume 30, numéro 1, 2006

Problématiques des sexes

Gender issues

URI : https://id.erudit.org/iderudit/016160ar

DOI : https://doi.org/10.7202/016160ar

Aller au sommaire du numéro

\section{Éditeur(s)}

Association Inuksiutiit Katimajiit Inc.

Centre interuniversitaire d'études et de recherches autochtones (CIÉRA)

\section{ISSN}

0701-1008 (imprimé)

1708-5268 (numérique)

Découvrir la revue

Citer ce compte rendu

Plumet, P. (2006). Compte rendu de [HOFFECKER, John F., 2005 A prehistory of the North. Human settlement of the higher latitude. Foreword by Brian Fagan, Rutgers University Press, New Brunswick, New Jersey, and London, 225 pages.] Études/Inuit/Studies, 30(1), 192-194. https://doi.org/10.7202/016160ar d'utilisation que vous pouvez consulter en ligne. 
the Prince of Wales Northern Heritage Centre, Yellowknife. These first-hand reports are invaluable for East Arctic kayak researchers and Arima is to be commended for bringing them to our attention. Arima's final section is an interesting array of archaeological evidence on the roots of arctic kayak design. He correctly claims no conclusions - the section is identified as "Speculations on Design Ancestry"-but the evidence is intriguing. This section brings us full circle back to John Heath's essay and his observations on the origins of kayak design.

This book is a very mixed collection of essays. In the book's all-too-brief introduction, Arima claims that the work is "[...] a comparative study of kayaks that focuses on historic development, design and construction and techniques" (p. ix). This implies a wholeness that is simply lacking. On my first reading, I found the eclectic nature of the content to be bewildering. On a more thorough reading, I found each of the articles to be of value, each in its own way. I was surprised to find such a brief and relatively unimportant essay by the doyen of Greenland kayak experts, H.C. Petersen. Surely something of greater depth could have been pulled from his writings. The book also needs a concluding section. There is minimal tie-in between the Greenland and East Arctic sections or the essays themselves. This could/should have been addressed. Uneven as this collection of essays is in subject and approach, it will interest any kayak aficionado. For the recreational paddler, the sections dealing with technique will be useful. For the historian/researcher, there is both old information to review and new to consider. The book is copiously illustrated with drawings, photographs and maps.

David W. Zimmerly 344 Lakewood Rd., RR3 Perth, Ontario K7H 3C5 Canada dwzimmerly@arctickayaks.com

HOFFECKER, John F.

2005 A prehistory of the North. Human settlement of the higher latitude. Foreword by Brian Fagan, Rutgers University Press, New Brunswick, New Jersey, and London, 225 pages.

Voici un ouvrage de synthèse sur la préhistoire du Grand Nord rédigé par un préhistorien états-unien qui a beaucoup travaillé en Europe centrale, dans le Caucase, mais aussi en Alaska et, par extension, sur l'environnement de la Béringie et l'adaptation de l'humanité aux régions froides. Le fait qu'il soit l'œuvre d'un seul auteur distingue ce livre des trop nombreux recueils qui réunissent les contributions de divers spécialistes autour d'un thème commun au profit de la variété des approches mais au détriment de l'homogénéité et de la cohérence du traitement. La préface souligne la nouveauté de cette entreprise en ce qui concerne le Grand Nord, ce qui est sans doute vrai, du moins en anglais.

Hoffecker a choisi de présenter son sujet par le biais d'un très bref résumé, un peu simplificateur, de l'expansion viking vers l'Arctique et de la première rencontre entre 
Européens et Inuit en insistant sur la supériorité adaptative des derniers. Ensuite, il retrace la progression de l'humanité vers les régions les plus froides du globe, depuis sa sortie d'Afrique. Pour cela, il suit de façon intéressante les grandes étapes de l'évolution anatomique et technologique de l'Homme en relation avec le climat. L'Europe tient une place importante, avec ses plus anciens sites découverts récemment en Italie et dans la péninsule Ibérique et ceux du Paléolithique inférieur, connus depuis plus longtemps, puis avec le Néandertal et, bien sûr, le Paléolithique supérieur qui constitue la pièce maîtresse. Les deux chapitres concernant l'humanité moderne dans le Nord et sa pénétration dans l'Arctique occupent plus de $35 \%$ du livre, notes et bibliographie exclues. Toutefois, le dernier chapitre, relativement bref bien que consacré aux peuples de la zone circumpolaire (15\% du texte), traite à lui tout seul de la colonisation de l'Arctique européen à partir de la toute fin du Paléolithique, du Néolithique sibérien et de l'expansion paléoesquimaude et néoesquimaude dans l'Arctique américain et au Groenland, ce qui est beaucoup en si peu de pages. Seules 13 pages, soit $9 \%$, sont consacrées à la période «esquimaude», certes beaucoup plus brève que le Paléolithique mais qui représente l'aboutissement de cette évolution.

L'essentiel du livre concerne donc l'Eurasie paléolithique, après une partie assez longue et détaillée sur l'expansion de l'humanité hors d'Afrique ainsi que les transformations qu'elle a connues jusqu'à l'Homme moderne. Les comparaisons des vestiges anatomiques humains d'Afrique et d'Europe sont faciles à comprendre pour le profane. Cette expansion et ces transformations sont divisées, pour la clarté et la simplification de l'exposé, en cinq stades, schématisés sur une carte (p. 8). Le stade 3 , cependant, ne tient pas compte des incursions probables de Néandertaliens vers le Nord, qui semblent pourtant attestées par des sites du Paléolithique moyen jusqu'au nord-est de l'Oural (Kroutaya Gora par exemple) et même en Finlande (Susiluola, récemment découvert). Les relations entre l'environnement, particulièrement le climat, et les cultures sont largement expliquées de façon simple et les différences entre la Sibérie et l'Europe sont soulignées avec pertinence. L'importance de la contribution du langage, puis du symbole et de la musique dans le processus d'adaptation culturelle aux contraintes des régions froides, ainsi que le lien entre langage, représentation du monde et technologie sont longuement exposés. L'auteur retrace l'apparition du ski, celle des patins de traîneaux, à Vis I en particulier, ainsi que les signes d'adaptation anatomique au froid chez l'homme moderne, observés sur les squelettes provenant de cimetières postglaciaires du nord de l'Europe.

Au lieu d'utiliser le repère habituel qu'offre la transition du Pléistocène à l'Holocène, vers 12 - $10 \mathrm{Ka}$, qu'il considère comme arbitraire, et qui correspond en gros à la transition entre le Paléolithique et le Mésolithique, Hoffecker souligne la continuité d'une période s'étendant de $20 \mathrm{Ka}$ à $7 \mathrm{Ka}$, caractérisée par de continuels ajustements climatiques et culturels. C'est après 7 Ka que l'ensemble de l'Arctique, audelà de l'Alaska et jusqu'au Groenland, est suffisamment libéré des glaciers pour permettre la fin de la colonisation humaine circumarctique. Le lecteur ne peut que regretter le peu de pages consacrées à cette dernière étape.

Ce livre s'adresse à un public large, mais déjà sensibilisé à la préhistoire et en particulier aux étudiants dans cette discipline qui apprécieront cette large synthèse, 
même si elle est un peu frustrante en raison de ses lacunes. Les illustrations photographiques sont malheureusement de mauvaise qualité et les cartes un peu trop schématiques. La bibliographie témoigne d'une certaine méconnaissance de la littérature européenne francophone, pourtant abondante et importante en ce qui concerne le Paléolithique.

Patrick Plumet 14, allée des Délieuses F78430 Louveciennes France plumet.patrick@free.fr

HURET, Pauline (dir.)

2003 Les Inuit de l'Arctique canadien, Québec, CIDEF-AFI, Collection Francophonie, 266 pages.

Depuis le succès international du film de Zacharias Kunuk, Atanarjuat. La légende de l'homme rapide, les Inuit et leur mode de vie provoquent la fascination. Plusieurs documentaires, articles et livres destinés à un auditoire curieux d'en savoir davantage ont été produits. L'ouvrage collectif intitulé Les Inuit de l'Arctique canadien, dont les textes ont été recueillis par Pauline Huret, s'adresse à la portion francophone de ce public. C'est d'ailleurs, à notre connaissance, la première fois qu'une introduction aussi complète est publiée dans la langue de Molière. C'est donc dans cet esprit qu'il faut aborder cette publication et se demander en quoi elle peut à la fois plaire à un large public et démystifier une réalité qui nous est souvent présentée de façon romantique.

Ce collectif s'ouvre sur une préface signée Michèle Therrien, professeure d'inuktitut et de culture inuit à l'Institut national des langues et civilisation orientales (Inalco) de Paris. Cette préface est suivie de portraits statistiques du Nunavik et du Nunavut. Vient ensuite l'introduction, qui comprend trois textes. Le premier, de l'anthropologue Yvon Csonka, présente l'histoire de l'Arctique central et oriental canadien de la préhistoire au $21^{\mathrm{e}}$ siècle. L'auteur réussit très bien à dresser de façon succincte l'historique du peuplement de cette région. Il termine avec une explication pertinente et indispensable des différences entre le rapport au temps chez les Inuit et chez les historiens occidentaux, ces derniers étant souvent déconcertés par le fait que les Inuit ne datent pas nécessairement les événements. La géographe Béatrice Collignon, quant à elle, explique en quoi la géographie dite savante n'arrive pas à appréhender le territoire inuit d'une façon adéquate et introduit les lecteurs aux perspectives inuit. Vient ensuite l'article de la linguiste Nicole Tersis, à propos de l'inuktitut. L'auteure dresse un historique de l'introduction du syllabaire chez les Inuit puis donne un bref aperçu de la structure syntaxique de l'inuktitut.

La partie suivante porte sur les systèmes de représentations inuit. Elle est également composée de trois textes. Le premier est signé par l'anthropologue Frédéric Laugrand qui présente au lecteur les cosmologies inuit. L'auteur ne se contente pas de dresser un portrait du chamanisme inuit tel qu'il était pratiqué avant l'arrivée des Euro- 